\title{
Detailed analysis of scatter contribution from different simulated geometries of $\mathrm{X}$-ray detectors
}

\author{
Elena Marimon ${ }^{1,2^{*}}$, Hammadi Nait-Charif ${ }^{1}$, Asmar Khan $^{2}$, Philip A. Marsden ${ }^{3}$, Oliver \\ Diaz $^{4}$ \\ ${ }^{1}$ Centre for Digital Entertainment, University of Bournemouth, Bournemouth, UK \\ \{Elena.MarimonMunoz, hncharif\} @bournemouth.ac.uk \\ ${ }^{2}$ Dexela Ltd, PerkinElmer, London, UK \\ \{Elena.Munoz, Asmar.khan\} @perkinelmer.com \\ ${ }^{3}$ Unitive Design, London, UK \\ phil@unitivedesign.co.uk \\ ${ }^{4}$ ViCOROB research institute, University of Girona, Girona, Spain \\ oliver.diaz@udg.edu
}

\begin{abstract}
Scattering is one of the main issues left in planar mammography examinations, as it degrades the quality of the image and complicates the diagnostic process. Although widely used, anti-scatter grids have been found to be inefficient, increasing the dose delivered, the equipment price and not eliminating all the scattered radiation. Alternative scattering reduction methods, based on postprocessing algorithms using Monte Carlo (MC) simulations, are being developed to substitute anti-scatter grids. Idealized detectors are commonly used in the simulations for the purpose of simplification. In this study, the scatter distribution of three detector geometries is analyzed and compared: Case 1 makes use of idealized detector geometry, Case 2 uses a scintillator plate and Case 3 uses a more realistic detector simulation, based on the structure of an indirect mammography $\mathrm{X}$-ray detector. This paper demonstrates that common configuration simplifications may introduce up to $14 \%$ of underestimation of the scatter in simulation results.
\end{abstract}

Keywords: Digital mammography, scatter, post-processing, Monte Carlo, X-ray detector.

\section{Introduction.}

Scattered radiation remains one of the main challenges in digital mammography [1], limiting the quantitative usefulness of radiographic images. It reduces the quality of the image, degrades the contrast and the signal to noise ratio, reduces the dynamic range and therefore affects the diagnosis of low contrast lesions and small microcalcifications $[2,3]$.

At present, the most widespread technique to reduce the scattered radiation in mammography, makes use of anti-scatter grids. However, anti-scatter grids are an incomplete solution, adding complexity and cost to the mammography system manufacturing. Although they help to improve image quality, they also attenuate primary radiation, 
leading to an increase in the patient dose delivered (up to a factor of 3 ) to maintain a constant Detector Air Kerma (DAK) [1], [4, 5].

The limitations of the anti-scatter grids have contributed to the emergence of new scatter reduction methods, based on image post-processing. Although scatter can be estimated using Monte Carlo (MC) simulations, faster and more flexible methods, such as the convolution-based scatter estimation method, have lately become more attractive $[4],[6,7]$. This method is based on the idea that the scatter in the system is spatially diffuse, thus it can be approximated by a two-dimensional low-pass convolution filter of the primary image [3]. In this paper, we study the scatter contribution of different detector geometries and their influence on the simulated filters, i.e. point spread function (PSF) kernels, in order to study possible simplifications in future scatter modelling.

\section{Methodology}

The image recorded in a digital mammography detector is the combination of energy deposited by primary and scattered X-ray photons. In this work, the contribution of the detector geometry to the final scatter PSF (SPSF) kernels will be analyzed.

\subsection{Software used}

MC simulations, based on the GEANT4 toolkit (version 10.01.p02), were used to study the production of scattered radiation in the simulated mammography geometry. GEANT4 is a widely used toolkit for simulating physical processes, including those occurring in mammography $[8,9,10]$.

\subsection{Scatter Point Spread Function}

SPSF kernels are obtained from the MC simulation using a narrow X-ray beam which is represented by a normally-incident two-dimensional spatial delta function, i.e. the $\mathrm{X}$ ray beam is simulated following the narrow pencil beam method [2], [7], [10]. Energy from scattered radiation ( $\mathrm{S}$ ) was binned into $1 \mathrm{~mm}$ radius (r) concentric annuli (up to $100 \mathrm{~mm})$. These were then normalized to the primary image $\mathrm{P}(0)$ to create SPSFs. These SPSFs were also normalized by the area of the annuli, A(r), as described in [11].

$$
\operatorname{SPSF}(r)=\frac{S(r)}{P(0) A(r)}\left[\mathrm{mm}^{-2}\right]
$$

\subsection{Validation Geometries}

Before the simulations were performed, the scatter PSF code was validated using, as a benchmark, the values given in the report of the American Association of Physicists in medicine (AAPM) task group 195, case 3 - Mammography and breast tomosynthesis $[12,13]$.

The geometry used for the validation included breast compression and support paddles, both made of PMMA and $2 \mathrm{~mm}$ thick, a semi-circular cylinder as breast phantom 
- $46 \mathrm{~mm}$ thick, composed by $80 / 20 \%$ of adipose/glandular tissue and surrounded by a $2 \mathrm{~mm}$ thick skin layer - and a $13 \mathrm{~mm}$ air gap between the support paddle and an idealized detector surface. The Source to Image Distance (SID) was $660 \mathrm{~mm}$. A patient body made of water was also included, adjacent to the breast phantom's chest wall side and centered in the vertical direction with the breast phantom. Fig.1-A shows a diagram of the geometry $[12,13]$.

A combination of two X-ray beams (cone and pencil beam) and two X-ray spectra (monoenergetic and polyenergetic) were used for the validation, see Table 1 . The spectrum values were obtained from [12]. For each of them, the primary radiation, Compton scattering, Rayleigh scattering and multiple scattering were measured in 7 different regions of interest (ROIs), as described by [12]. Enough particles to produce Standard Error of the Mean (SEM) values equal or lower than $1 \%$ were run. The maximum discrepancies were obtained when recording the multiple scattering values.

Table 1. The table shows the four X-ray source/spectrum combinations used for the validation of the scatter PSF code. The last column shows the maximum discrepancy found when comparing the results with the AAPM report - group 195- Case 3 data [12].

\begin{tabular}{cccc}
\hline Validation & Source type & Spectrum & $\begin{array}{c}\text { AAPM comparison (\%) } \\
\text { Average (Maximum) }\end{array}$ \\
\hline V1 & Cone Beam & Mono energetic $(16.8 \mathrm{keV})$ & $0.82(4.0)$ \\
V2 & Cone Beam & $\begin{array}{c}30 \mathrm{kVp} \mathrm{Mo} / \mathrm{Mo} \\
(\mathrm{HVL}=0.3431 \mathrm{mmAl})\end{array}$ & $0.48(3.6)$ \\
& & Mono energetic $(16.8 \mathrm{keV})$ & $0.37(1.6)$ \\
V3 & Pencil Beam & $0.29(1.9)$ \\
V4 & Pencil Beam & $30 \mathrm{kVp} \mathrm{Mo} / \mathrm{Mo}$ & \\
& & $(\mathrm{HVL}=0.3431 \mathrm{mmAl})$ & \\
\hline
\end{tabular}

\subsection{Geometry used in this study}

The mammography geometry used was slightly modified from the one suggested in the AAPM-group 195 report, [12], to adjust it to the needs of this study. Firstly the patient body was not included in the simulation, as we were only interested in studying the scattering produced in the center of the breast, and secondly the D-shaped phantom was changed to a cylinder-shaped phantom, Fig. 1-B shows an example of this geometry.

In this report, the SPSF kernels are simulated using a narrow pencil beam and spectrum of $30 \mathrm{kVp} \mathrm{Mo} / \mathrm{Mo}(\mathrm{HVL}=0.3431 \mathrm{mmAl})$. The photon cross-section "Electromagnetic physics option 4 package" was used both for the validation and the experiment. 20 to 60 runs, of $10^{9} \mathrm{X}$-ray photons each, were simulated, ensuring uncertainties lower than $1.5 \%$. All presented values are represented as:

$$
q \pm 3 s
$$

where, $\bar{q}$ is the mean of the value under study and s represents the uncertainty. s were calculated following equation (3), as suggested by Sempau et al.[14]: 


$$
S=\sqrt{\frac{1}{N}\left(\frac{\sum_{i} q_{i}^{2}}{N}-\bar{q}^{2}\right)}
$$

where, $\mathrm{N}$ is the number of runs and $\mathrm{q}_{\mathrm{i}}$ is the value under study for run $\mathrm{N}=\mathrm{i}$.

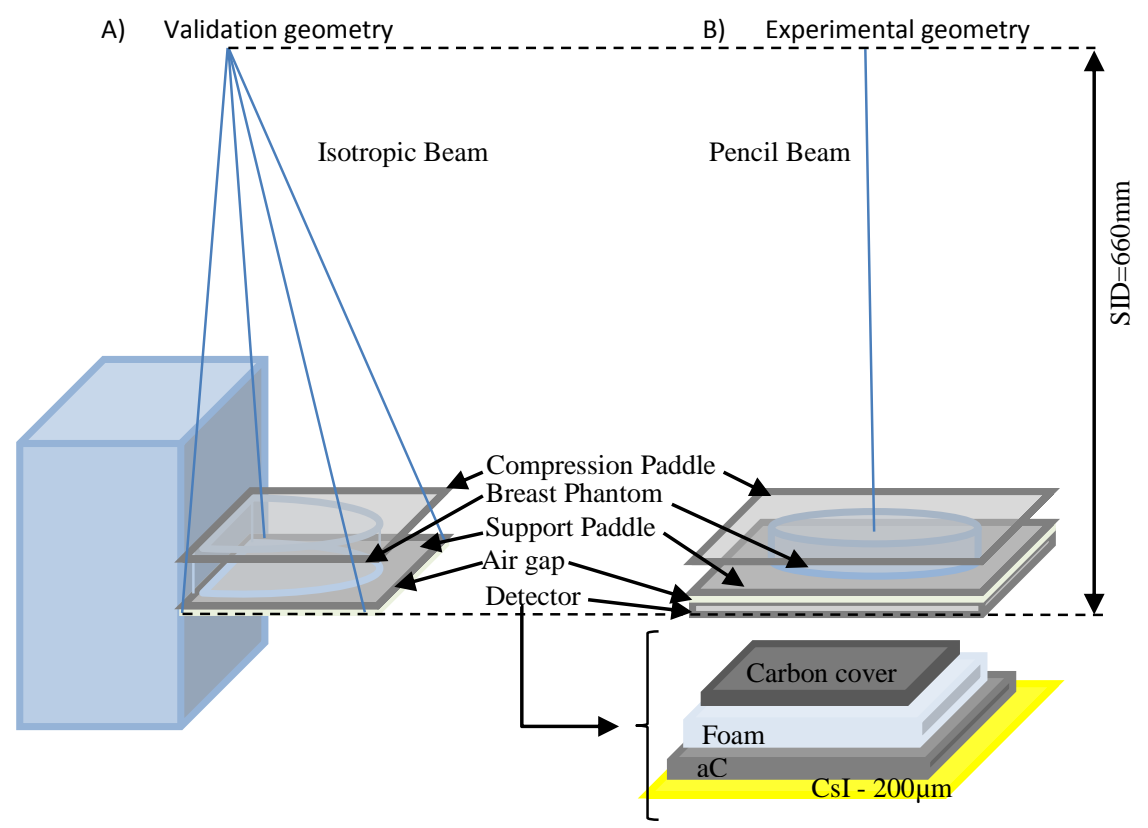

Fig. 1. Diagram of the geometries used for validation purposes (A) and for the experiments (B). Figure A shows an example using a cone beam aligned with the chest wall of the breast phantom. Figure B shows the pencil beam example and a detector added after the air gap.

\subsection{Experiment and analysis}

To evaluate the contribution of the detector geometry to the simulated PSF, three different detector setups were compared:

1. Case 1 - Ideal detector geometry: The detector is assumed to be an ideal X-ray sensitive surface. The energy stored comes from all X-ray photons that reach the detector surface.

2. Case 2 - Simplified detector geometry: The detector is assumed to be only the scintillator plate $(200 \mu \mathrm{m}$ CsI on a $1.5 \mathrm{~mm}$ of amorphous Carbon (aC) substrate [15]), placed right after an air gap of $13 \mathrm{~mm}$.

3. Case 3 - Realistic detector geometry: The different layers of the detector down to the scintillator surface are considered and their contribution studied. A carbon cover was placed after the $13 \mathrm{~mm}$ air gap. Then, a second air gap was added between the cover and the Case 2 scintillator plane. The second air gap is an approximation used to simulate a piece of foam, see Fig. 2. The data was obtained via private communication with PerkinElmer Inc. 
Case 2 simplification is often found in the literature, in cases where the detector structure is unknown. To evaluate how effective this simplification is, Case 3 simulates a more realistic detector structure. The interactions occurring after the X-rays are scintillated were not taken into account.

For the three detector geometries described above, three breast thicknesses and three breast glandularity percentages - material composition obtained from Hammerstein et al. [16] - were analyzed. Table 2 shows all of the combinations under study. Data from experiment A are taken as the reference values. Radial symmetry was assumed in this study, since the pencil beam hits a location far from the edges of the simulated phantom.

Table 2. The table shows the characteristics of the different experiments that have been studied. It specifies the breast thickness and glandularity of the phantom

\begin{tabular}{cccc}
\hline Exp. & Breast Thickness $(\mathrm{mm})$ & Glandularity $(\%)$ & $\begin{array}{c}\text { Detector geometry: } \\
\text { Cases } 1,2,3\end{array}$ \\
\hline A & 50 & 20 & A.1, A.2, A.3 \\
B & 30 & 20 & B.1, B.2, B.3 \\
C & 80 & 20 & C.1, C.2, C.3 \\
D & 50 & 35 & D.1, D.2, D.3 \\
E & 50 & 50 & E.1, E.2, E.3 \\
\hline
\end{tabular}

The figure of merit used for the analysis of the result was the total SPR, SPR (4), which corresponds to the area under the SPSF' curve and represents the amount of total scatter.

$$
\begin{gathered}
S P R_{T}=\int_{r=0}^{r=r_{\text {max }}} \operatorname{SPSF}^{\prime}(r) d r \\
\operatorname{SPSF}^{\prime}(r)=\operatorname{SPSF}(r) A(r)
\end{gathered}
$$

\section{$3 \quad$ Results}

\subsection{Comparison of the scatter kernels obtained for each geometry}

Fig. 2 shows the SPSF(r) for cases A.1, A.2 and A.3. The figure shows the spatial distribution of the scatter as a function of radial distance. The plot on top of Fig. 2 is used to highlight the differences between the cases, using results from A.1 as reference.

\subsection{Comparison of the SPRT obtained for each experiment}

The area under the SPSF(r) curve, i.e. SPR , allows the comparison of the total scatter contribution of the different experiments, see Table 3. The table also shows the ratio between cases 1 and 3 and cases 2 and 3, giving an estimation of the change in the scattering between cases and experiments. The values were calculated for a radial distance of $100 \mathrm{~mm}$. 


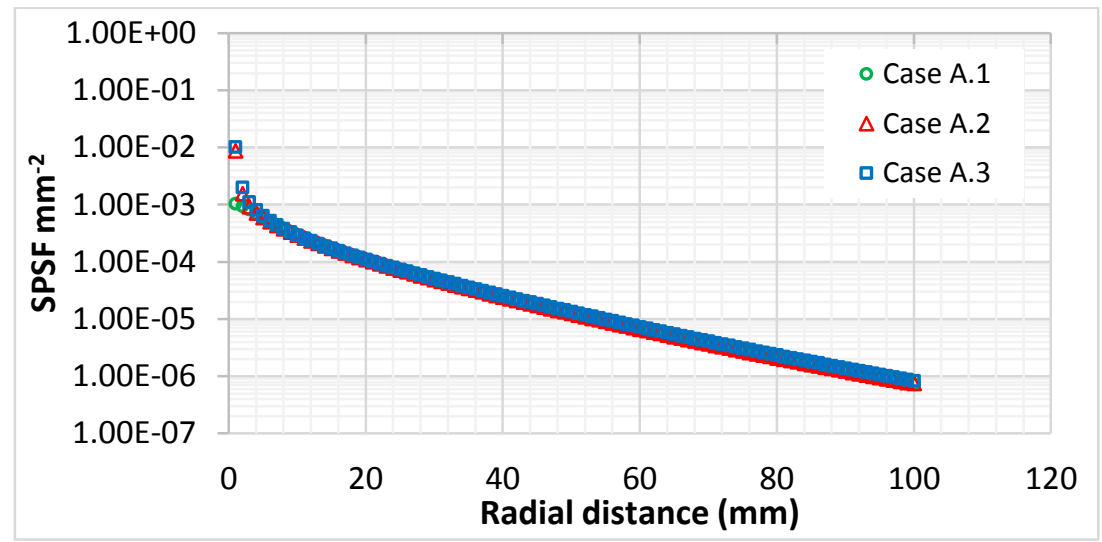

Fig. 2. The plot shows the SPSF(r) curves obtained when using an ideal detector (Case A.1), a scintillator plate (Case A.2) and a realistic detector geometry (Case A.3).

Table 3. The table shows the average $\mathrm{SPR}_{\mathrm{T}}$ values and their uncertainty $(3 \mathrm{~s}, \%)$ for the three detector geometries and five experiments run (A-E) and the ratios between $\mathrm{C} 1-\mathrm{C} 3$ and $\mathrm{C} 2-\mathrm{C} 3$.

\begin{tabular}{ccccrr}
\hline & \multicolumn{3}{c}{ Total SPR (Error in \%) } & & \\
\cline { 2 - 4 } Exp. & Case 1 & Case 2 & Case 3 & C1/C3 & C2/C3 \\
\hline A & $0.60(0.04)$ & $0.64(0.04)$ & $0.67(0.04)$ & 0.90 & 0.95 \\
B & $0.40(0.04)$ & $0.43(0.04)$ & $0.46(0.04)$ & 0.86 & 0.94 \\
C & $0.92(0.13)$ & $0.97(0.14)$ & $0.10(0.14)$ & 0.92 & 0.97 \\
D & $0.61(0.07)$ & $0.65(0.08)$ & $0.68(0.08)$ & 0.90 & 0.95 \\
E & $0.62(0.08)$ & $0.66(0.08)$ & $0.69(0.08)$ & 0.90 & 0.95 \\
\hline
\end{tabular}

Looking at the last two columns of Table 3, the ratio of $\mathrm{C} 1$ or $\mathrm{C} 2$ with respect $\mathrm{C} 3$ can be seen in Fig. 3 (expressed in \%).

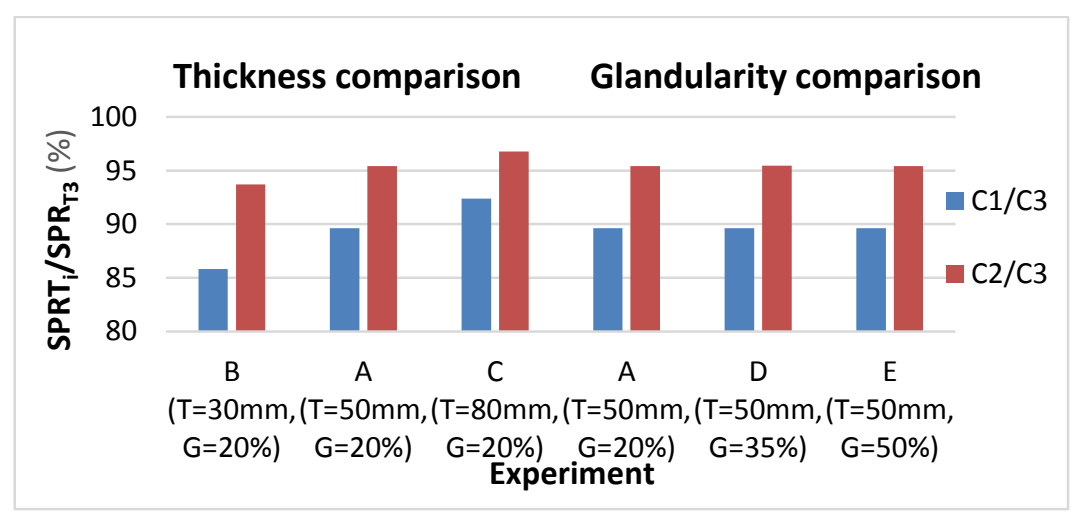

Fig. 3. The plot shows the ratio between the ideal detector geometry $\mathrm{C} 1$ (and the scintillator plate geometry $\mathrm{C} 2$ ) and the more realistic geometry (C3) for different breast thickness (T) and glandularity $(\mathrm{G})$ combinations. 


\section{Discussion and Conclusions}

Convolution-based scattering removal methods have been suggested in the literature for scattering reduction in mammography as an alternative to the anti-scatter grids [3], $[6,7]$. This method makes use of simplified MC simulations for kernel (PSF) calculations, which are used to post-process acquired images.

A realistic mammography geometry is required for the PSF simulations, to account for scatter from elements such as compression paddles and breast support which can have a large contribution in the final image [10], [17]. This work focuses on the contribution of the detector geometry to the SPRs. To that end, three geometries have been studied for three different glandularities and thicknesses: an ideal case where the detector does not contribute to the scatter (Case 1), an intermediate case where the detector is approximated by a scintillator plate (Case 2) and a more realistic detector structure (Case 3).

Fig. 2 shows a plot of the SPSF as a function of the radial distance. In the graph, it is possible to see that cases 2 and 3 show a peak at shorter radial distances, up to $4 \mathrm{~mm}$, that is not present in Case 1. This is in line with previous observation of Diaz [11].

Considering the overall scatter contribution (see Table 3), the total amount of scatter increases with additional layers of material. As expected, a more complex detector geometry presents higher scatter to primary ratio values. If Case 1 and Case 3 are compared, a discrepancy between $10-14 \%$ is found for the different experiments, while a discrepancy between 3-6\% can be seen when comparing cases 2 and 3 . These results show that it is important to add the detector information into the simulations. If the dimensions and component materials are unknown, simply adding the scintillator (substrate and CsI:Tl) to the geometry can improve the scatter simulation by $5-8 \%$, when compared with the detailed detector geometry, Case3.

The changes in the ratio between the cases, as a function of variations in the glandularity or the thickness of the breast, can be seen in Fig. 3. The figure shows the percentage discrepancy from the reference experiment. The results show that Case 1 is more affected by the phantom changes than Case 2 .

For thinner breasts (experiment B), the variation with Case 3 is higher, so the importance of including the detector details in the simulation increases. As thicker breast phantoms have greater contribution to the total SPR, we believe that the relative contribution from the detector geometry to the total SPR is decreased.

Glandularity variations do not seem to have a considerable effect on the ratio between the cases. This is in line with the literature, [10], [18].

Acknowledgements: This work has been done in collaboration with PerkinElmer. The authors would like to thank FilmLight Ltd for their donation of computer resources.

Oliver Díaz is supported by the European Union within the Marie Sklodowska-Curie Innovative Training Networks (H2020-MSCA-IF-2014 SCARtool project, reference 657875) and the Ministry of Economy and Competitiveness of Spain, under project reference DPI2015-68442-R. 


\section{References}

1. A. Wang et al., "Asymmetric Scatter Kernels for Software-Based Scatter Correction of Gridless Mammography”, SPIE Medical Imaging, vol. 9412, pp.1-1-7, 2015.

2. J. M. Boone and V. N. Cooper, "Scatter/primary in mammography: Monte Carlo validation", vol. 27 (8), 2000

3. J. L. Ducote and S. Molloi, "Scatter correction in digital mammography based on image deconvolution", IOP - Physics in Medicine and Biology, vol. 55, pp. 1295-1309, 2010.

4. J. Binst et al., "Evaluation of automated CDMAM readings for non-standard CDMAM imaging conditions: Grid-less acquisitions and scatter correction", Oxford University Press - Radiation Protection Dosimetry, pp. 1-4, 2015.

5. A. Krol et al., "Scatter reduction in mammography with air gap", Medical Physics, vol. 23, no. 7, pp. 1263-1270, 1996.

6. O. Diaz, et al., "A fast scatter field estimation for digital breast tomosynthesis", Proc. SPIE Medical Imaging, vol. 8313, 2012.

7. O. Diaz, et al., "Estimation of scattered radiation in digital breast tomosynthesis", IOP Physics in Medicine and Biology, vol. 59, pp. 4375-4390, 2014.

8. J. Allison, et al., "GEANT4 developments and applications", IEEE Transactions on nuclear science, vol. 33, no. 1, pp. 270-278, 2006.

9. P. V. Feijó and G. Hoff, "GEANT4 Validation on Mammography Applications", IEEE Nuclear Science Symposium, 2008.

10. I. Sechopoulos, et al., "Scatter radiation in digital tomosynthesis of the breast", Medical Physics, vol. 34, no. 2, pp. 564-576, 2007.

11. O. Diaz, Scattered radiation in projection X-ray mammography and digital breast tomosynthesis. PhD Thesis, University of Surrey, 2013.

12. I. Sechopoulos, et al., "The report of AAPM Task Group 195: Monte Carlo Reference Data Sets for Imaging Research", AAPM, 2015.

13. I. Sechopoulos, et al., "Monte Carlo reference data sets for imaging research: Executive summary of the report of AAPM Research Committee Task Group 195", Medical Physics, vol. 42, p.5679, 2015.

14. J. Sempau, A. Sánchez-Reyes, F. Salvat, et al., "Monte Carlo simulation of electron beams from an accelerator head using PENELOPE", Physics in medicine and biology, vol. 46, no. 4, pp. 1163-1186, 2001.

15. Hamamatsu, "Scintillator plates - Hamamatsu photonics", Hamamatsu photonics, [Online]. Available: http://www.hamamatsu.com/jp/en/product/category/3100/3010/index.html. [Accessed 23 March 2016].

16. G. R. Hammerstein, D. W. Miller, D. R. White, M. E. Masterson, Q. J. Woodard and J. S. Laughlin, "Absorbed radiation dose in mammography", Radiology, vol. 130, pp. 485-491, 1979.

17. D. R. Dance and G. J. Day, "The computation of scatter in mammography by Monte Carlo methods", Physics in Medicine and Biology, vol. 29, pp. 237-247, 1984.

18. J. Boone, et al., "Scatter/primary in mammography: Comprehensive results", Medical Physics, vol. 27, no. 10, pp. 2408-2416, 2000. 